\title{
Beichte und Buße im ökumenischen Gespräch
}

\author{
Ein Beispiel für die bleibende theologische Bedeutung der \\ Confessio Augustana
}

\section{Eine These und die gewählte Form ihrer Begründung}

Ich beginne mit einer These: Die Prägnanz der von Philipp Melanchthon in der Confessio Augustana (künftig CA) formulierten Argumente ist eine bleibende Herausforderung für die Theologien aller Konfessionen jeder Zeit, sich den Fragen $\mathrm{zu}$ stellen, die in diesem Dokument angesprochen werden. Die formulierte These setzt die Kenntnis der theologischen Reflexionen im 16. Jahrhundert voraus und lässt zugleich eine Bezugnahme auf die Gegenwart erwarten. In dem Geschehen der Kommunikation zwischen früheren Erkenntnissen und heutigen Einsichten sind die in den ökumenischen Gesprächen inzwischen gewonnenen Konvergenzen von hoher Bedeutung. Mit meiner These verbindet sich der Gedanke, dass die CA durch eine theologisch reflektierte Würdigung der in ihr formulierten Anliegen die ihr entsprechende christlich-ökumenische Anerkennung findet. Unter „Anerkennung"verstehe ich in meinem Beitrag - ganz schlicht - eine Würdigung der theologischen Argumentationen in Gestalt der kritischen Aufnahme der in der CA eingenommenen Positionierungen.

Die Begründung der These kann aus meiner Sicht angesichts der Fülle der in der CA besprochenen kontroverstheologischen Themen nur exemplarisch geschehen. Ich wähle beispielhaft eine Thematik, die mich von den ersten Zeiten des eigenen theologischen Wirkens an begleitet: ${ }^{1}$ das ökumenische Gespräch über

1 Vgl. Dorothea Sattler, Gelebte Buße. Das menschliche Bußwerk (satisfactio) im Ökumenischen Gespräch, Mainz 1992. In jüngerer Zeit habe ich mich insbesondere bei der Edition der bußtheologischen Schriften von Karl Rahner erneut mit der Thematik befasst: vgl. Karl Rahner, Mensch und Sünde. Schriften zur Geschichte und Theologie der Buße. Sämtliche Werke, Bd. 11, Freiburg i.B. 2005; ders., De Paenitentia I und II. Dogmatische Vorlesungen zum Bußsakrament. Sämtliche Werke, Bd. 6.1-6.2, Freiburg i.B. 2007/2009. Rahner hat sein umfassendes Wissen um die Geschichte der Buße mit seiner eigenen Perspektive verbunden, existentielle Zugänge zum

○ OpenAccess. ( 2022 Dorothea Sattler, publiziert von De Gruyter. (cc) BY-NC-ND Dieses Werk ist lizenziert unter einer Creative Commons Namensnennung - Nicht kommerziell - Keine Bearbeitung 4.0 International Lizenz. https://doi.org/10.1515/9783110683868-025 
Beichte und Buße, von dem ausgehend Grundfragen der Erlösungslehre mit anthropologischem Bezug zu besprechen sind. Fragen möchte ich, ob es aus gegenwärtiger Perspektive gelingen könnte, die theologischen Äußerungen in der CA mit der römisch-katholischen Tradition in ein solches Gespräch zu führen, an dessen (immer vorläufigem) Ende ein wechselseitiges Verstehen der gemeinsamen christlich motivierten Interessen möglich erscheint.

Im Folgenden werde ich zunächst (Teil 2) die Aussagen der CA über Beichte und Buße in ihrem zeitgeschichtlichen reformatorischen Kontext vorstellen (Abschnitt 2.1), an die römisch-katholische Gegenrede im 16. Jahrhundert erinnern (Abschnitt 2.2) und referieren, warum gerade diese Thematik als eine kaum lösbare Problematik in Publikationen um 1980 bei den Bemühungen um eine Anerkennung der CA nach 450 Jahren galt (Abschnitt 2.3); sodann (Teil 3) werde ich bezogen auf die theologischen Anliegen der CA im gewählten Themenkreis Überlegungen aus heutiger Sicht einbringen - konkret zur Bedeutung des Sündenbekenntnisses (Abschnitt 3.1) sowie zur Unterscheidung zwischen der inneren Gesinnung (Reue und Glaube) und einem äußeren Geschehen im kirchlichen Raum (Abschnitt 3.2). Ich schließe mit Reflexionen auf die bleibende theologische Bedeutung der CA angesichts der Herausforderungen in der christlichen Gegenwart (Teil 4).

\section{Aussagen zum Thema Beichte und Buße in der Confessio Augustana und deren Rezeption}

\subsection{Reformatorische Anliegen im Themenbereich Beichte und Buße}

„Steht die Bußlehre der Confessio Augustana einer Anerkennung durch die katholische Kirche im Wege?“2 - so fragte Hans Jorissen im Kontext der vielen Bemühungen um eine ökumenische Würdigung der CA in den 70er Jahren des

\footnotetext{
Verständnis der Sakramente zu erschließen. Darin stimmt er mit den reformatorischen Anliegen im Grundsatz überein.

2 Hans Jorissen, Steht die Bußlehre der Confessio Augustana einer Anerkennung durch die katholische Kirche im Wege?, in: Harding Meyer/Heinz Schütte/Hans-Joachim Mund (Hg.), Katholische Anerkennung des Augsburgischen Bekenntnisses? Ein Vorstoß zur Einheit zwischen katholischer und lutherischer Kirche, Frankfurt a. M. 1977, 132-150.
} 
20. Jahrhunderts. ${ }^{3}$ Vor dem Hintergrund der nach dem II. Vatikanischen Konzil auf kirchenamtlicher Ebene aufgenommenen Dialoge zwischen dem Lutherischen Weltbund und dem Päpstlichen Rat zur Förderung der Einheit der Christen war es sehr naheliegend, nach Lehrtexten Ausschau zu halten, die in den Konfessionen Autorität haben und als Grundlage der weiteren Gespräche gelten konnten. Die CA bot sich als Bezugstext an, da sie - anders als die inner-lutherische Konkordienformel von 1577 - noch weniger kontroverstheologisch geprägt war. Martin Luther selbst kann als Verfechter einer ökumenischen Würdigung der CA gelten; in einer Tischrede soll er gesagt haben: „Ich habe sorg, das wir nimer mehr so nahent zw samen khumen werden als zw Augspurg“4.

\subsubsection{Die theologische Argumentation in der Confessio Augustana}

In der theologischen Gesamtkomposition der CA gehören die Aussagen über Beichte und Buße zu den einundzwanzig Artikeln, bei denen Philipp Melanchthon in Anbetracht der biblischen Begründung der Lehren sowie der vielfachen Bezeugung in der christlichen Tradition optimistisch mutmaßte, dass , auch unser widdersacher können inn obangezeigten Artikeln nicht uneinig mit uns sein“"5. In argumentativ überzeugender Weise schließen die Ausführungen über die Beichte und Buße an das Bekenntnis zu Gottes Wesen (CA 1), die menschliche Sündigkeit (CA 2), die Erlösung in Christus Jesus (CA 3), die göttliche Entschiedenheit aus Gnade und Barmherzigkeit für die Rechtfertigung der Sünder und Sünderinnen (CA 4), Gottes Wirken in der Kirche durch den Dienst an der Verkündigung und der Feier der Sakramente (CA 5-8) an. Nach den kurzen Ausführungen über die Taufe (CA 9) und das Abendmahl (CA 10) folgen zwei Äußerungen über Beichte (CA 11) und Buße (CA 12), das rechte Verständnis der Wirkweise der Sakramente (CA 13) und das ordinierte Amt (CA 14). Die weiteren Überlegungen im ersten Teil der CA befassen sich gedanklich ebenfalls mit der rechten Ordnung des kirchlichen Handelns angesichts der Sündigkeit der Geschöpfe. Zunehmend wird dabei die eschatologische Dimension des christlichen Glaubens angesprochen - insbesondere in CA 17 und 21 im Blick auf die Erwartung des göttlichen Gerichts und die

3 Vgl. die Literatursammlung von Heiner Grote, Studien und Beiträge zur jüngsten AugustanaDebatte. Eine Bibliographie, in: Materialdienst des Konfessionskundlichen Instituts 29 (1978), $26-27$.

4 WA TR 4, 495.

5 CA 21: BSELK, 130, 17-18. Ich zitiere die CA nach folgender Ausgabe: Die Bekenntnisschriften der Evangelisch-Lutherischen Kirche. Vollständige Neuedition, hg.v. Irene Dingel, Göttingen 2014. 
Anrufung der bereits vollendeten Heiligen zur Fürsprache für die Sünderinnen und Sünder bei Gott.

Die Verortung der beiden Artikel der CA über das Thema Beichte und Buße im größeren Kontext der reformatorischen Lehre von den Sakramenten war vielfach Anlass zu Kommentierungen. Offenkundig war Philipp Melanchthon daran gelegen, die biblisch überlieferte Weisung Jesu Christi, die Vergebung der Sünden als eine von Gott gebotene Zeichenhandlung zu verstehen, $\mathrm{zu}$ bewahren. Beichte und Buße sind aus reformatorischer Sicht geeignete kirchliche Handlungen, auf die bezogen sich exemplarisch erörtern lässt, wie Sakramente wirken: aufgrund der Gnade Gottes in einem zum Glauben berufenen Menschen.

Die konkreten Äußerungen der CA zur Beichte und zur Buße lassen sich in folgender Weise zusammenfassen: Die Praxis der Beichte möge bewahrt bleiben, ein detailliertes Bekenntnis aller einzelnen Sünden ist jedoch nicht erforderlich, um Gottes Erbarmen zu erlangen - ja, es ist sogar nicht einmal möglich (CA 11). Die Kirche darf niemandem, der oder die Reue zeigt und den christlichen Glauben bekennt, die Gabe einer zweiten Buße nach der Taufe verweigern (CA 12). Die rechte Buße besteht im eigenen Erschrecken über die Sünde, im Vertrauen auf die Barmherzigkeit Gottes und im Versprechen, sich zu bessern. Diese „Früchte der Buße“ - die guten Werke - sind als eine Folge des erlösenden Zuspruchs der Vergebung, der allein in Gottes Handeln durch Jesus Christus begründet ist, zu verstehen. In den wenigen Sätzen der CA zum Thema Beichte und Buße werden zentrale Anliegen der Reformation sehr deutlich: die Unterordnung des kirchlichen Handelns unter Gottes Verheißung seiner Gnade Sündern und Sünderinnen gegenüber sowie das Streben nach einer Übereinstimmung zwischen innerer Gesinnung in Reue und Glauben und äußerer Tat im Bekenntnis der Sünden und Werken der Liebe.

\subsubsection{Hintergründige Bezüge zum Leben und Denken von Martin Luther}

Martin Luther hat sich bis zu seinem Lebensende für die Bewahrung der Praxis der Einzelbeichte verbunden mit der Absolution durch einen ordinierten Amtsträger eingesetzt. ${ }^{6}$ Der in der Schule von Lukas Cranach 1547 (ein Jahr nach dem Tod von Luther 1546) vollendete und heute in der Stadtkirche von Wittenberg zu betrachtende Flügelaltar zum Gedächtnis des Verständnisses des Dienstes an der

6 Vgl. Ernst Bezzel, Frei zum Eingeständnis. Geschichte und Praxis der evangelischen Einzelbeichte, Stuttgart 1982; Martin Ohst, Pflichtbeichte. Untersuchungen zum Bußwesen im Hohen und Späten Mittelalter, Tübingen 1995. 
Verkündigung des Wortes Gottes und der Feier der Sakramente bei Luther bezeugt anschaulich, dass seine Zeitgenossen mit seiner Wertschätzung des Geschehens der Beichte vertraut waren: Die von der Predigtkanzel ausgehende Verkündigung des österlichen Evangeliums konkretisiert sich in drei Sakramenten, für die nach der neutestamentlichen Überlieferung Jesus Christus ein Wort der Stiftung hinterlassen hat: für die Taufe, für das Abendmahl und für die Absolution der mit Reue erfüllten gläubigen Christinnen und Christen.

In der Beschreibung der Geschichte theologischer Thesen ist es inzwischen vertraut, einzelne Erkenntnisse in ihren sozialen und biographischen Kontexten verstehen zu lernen. 1533 schrieb Martin Luther an die Gemeinde zu Frankfurt am Main: „Wenn tausend und abertausend Welt mein wäre, so wollt ich alles lieber verlieren, denn ich wollt von der Beichte das geringste Stücklein aus der Kirchen kommen lassen: ja, lieber sollt' mir sein des Papsttums Tyrannei von Fasten, Feiern, Kleidern [...], denn dass die Beicht sollt' von den Christen genommen werden; denn sie ist der Christen erste, nötigste und nützlichste Schule, darin sie lernen Gottes Wort und ihren Glauben verstehen und üben, welches sie nicht so gewaltig tun in öffentlichen Lektionen und Predigten"7. Martin Luther fühlte sich missverstanden mit seiner Kritik an der Beichte. Er kämpfte für den Erhalt dieses kostbaren Guts. Die Praxis sollte bleiben, nur erneuert musste sie aus seiner Sicht werden.

Damals wie heute galten Verweise auf Missbräuche, wie auch Martin Luther sie wagte, als Ermutigung zur Preisgabe des Sakramentes der Versöhnung überhaupt. Wie bei kaum einem anderen kirchlichen Geschehen ist bei dieser gottesdienstlichen Feier die Sensibilität aller Beteiligten gefordert. Verletzungen können sehr leicht entstehen. Die Abschaffung der gesamten Einrichtung erscheint dann schnell als die vordergründig einfachste Lösung. Doch wird damit ein hoher Preis gezahlt. Nach Martin Luther kann nicht einmal die Predigt, die Schriftverkündigung, so tief in das menschliche Innere reichen wie der Zuspruch der Sündenvergebung in der Absolution. Die Wortverkündigung hören alle zugleich in ganz unterschiedlichen Lebenssituationen. Das Wort der Lossprechung von der Last der Schuld trifft einen einzelnen Menschen in seiner unverwechselbaren Eigenheit. Es ist ein unverzichtbar kostbares Wort der Zusage des barmherzigen Gottes an den sündigen Menschen - so sah es Martin Luther. Um sich als solches Gehör verschaffen zu können, bedurfte es der Unterscheidung zwischen dem Evangelium gemäßen Sinn der Buße und geschichtlich bedingten Zuwächsen, die diesen verstellen.

Martin Luther hielt im Sinne von CA 14 auch daran fest, dass der Dienst der öffentlichen Wortverkündigung und der Feier der Sakramente Menschen vorzubehalten sei, die dazu in rechtmäßiger Weise ordiniert sind. Im Blick auf das Buß-

7 WA 30/3, 569. 
sakrament zeigte Luther Offenheit für mehrere Positionen: Als ein Teil der öffentlichen Verkündigung des Evangeliums blieb dieser Dienst auch in Wittenberg den ordinierten Pastoren - vorab Johannes Bugenhagen, einem offenkundig begnadeten Beichthörenden - vorbehalten. Zugleich erinnerte Luther an die Tradition der Laienbeichte: Jeder und jede Getaufte ist von Gott berufen, andere Menschen zu vergewissern, als Sünderinnen und Sünder aufgrund ihres vertrauenden Glaubens von Gott angenommen zu sein.

\subsection{Römisch-katholische Lehrentscheide des Trienter Konzils}

Die Bußtheologie des Trienter Konzils ist als eine zeitgeschichtlich geprägte, durch die reformatorische Theologie herausgeforderte Lehrgestalt zu verstehen, deren primäres Anliegen es war, die Legitimität der bestehenden Bußpraxis zu begründen und auf diese Weise deren Erhalt zu gewährleisten. Die nach langen Debatten verabschiedeten Canones über die Buße auf dem Konzil von Trient ${ }^{8}$ sprechen sich für die Sakramentalität dieser kirchlichen Handlung aus, bestimmen die materia der Buße (das von Menschen gestaltete äußere Zeichen) als Reue, Bekenntnis und Genugtuung, die als einzelne jeweils näher beschrieben werden, und gehen auf die Frage nach der amtlichen Schlüsselgewalt in Gestalt der priesterlichen Absolution ein. In dem erläuternden Lehrtext (der Doctrina), der den knappen Formulierungen in den Canones vorausgeht, kommt die pastorale Sorge der Trienter Konzilsväter zur Sprache. Dies zeigt sich beispielsweise in der Zustimmung zu der (zumindest auch) therapeutischen Wirksamkeit des Bußsakraments. So kommt in den Ausführungen über die zeitlichen Sündenstrafen und die ihnen entsprechende Bußauflage zur Sprache, dass der Weg zur Versöhnung so zu gestalten sei, dass die Menschen zukünftig vorsichtiger und wachsamer sind; zudem sollen die leidvollen Folgen der Sünde durch tätige Buße geheilt werden. Das Bußwerk soll eine Gestalt haben, die das neue Leben schützt und als eine Arznei für die Schwachheit der Menschen erfahrbar ist. ${ }^{9}$

Im Vergleich mit den in der CA begründeten Lehrmeinungen sind insbesondere zwei Canones in den kontroverstheologischen Fokus geraten. Canon 4 der Trienter Bußdekrets lehrt über die drei Formen der Beteiligung des Menschen am Geschehen der sakramentalen Buße:

8 Vgl. die von Peter Hünermann auf der Grundlage der Sammlung von Heinrich Denzinger herausgegebene Edition: Kompendium der Glaubensbekenntnisse und Kirchlichen Lehrentscheide (künftig: DH), Freiburg i.B. 1991, hier DH, 1701-1715.

9 Vgl. DH, 1690 und 1691. 
Wer leugnet, dass zur vollständigen und vollkommenen Vergebung der Sünden drei Akte beim Büßenden gleichsam als Materie des Bußsakramentes erforderlich sind, nämlich die Reue, das Bekenntnis und die Genugtuung, welche die drei Bestandteile der Buße genannt werden; oder sagt, es gebe nur zwei Bestandteile der Buße, nämlich die dem Gewissen nach Erkenntnis der Sünde eingejagten Schrecken und der aufgrund des Evangeliums oder der Lossprechung empfangene Glaube, mit dem man glaubt, dass einem durch Christus die Sünden vergeben sind: der sei mit dem Anathema belegt ${ }^{10}$.

Ein weiterer Widerspruch zwischen der CA und der Trienter Lehre bezieht sich auf Canon 7:

Wer sagt, beim Sakrament der Buße sei es zur Vergebung der Sünden nicht nach göttlichem Recht notwendig, die Todsünden samt und sonders zu bekennen, an die man sich nach gehöriger und sorgfältiger vorheriger Überlegung erinnert, auch die verborgenen und diejenigen, die gegen die zwei letzten Vorschriften der Zehn Gebote gerichtet sind, mitsamt den Umständen, die die Art der Sünde verändern; sondern dieses Bekenntnis sei nur zur Erziehung und Tröstung des Büßenden nützlich und sei einst nur in Gebrauch gewesen, um eine kanonische Genugtuung aufzuerlegen; oder sagt, wer alle Sünden zu bekennen trachte, wolle der göttlichen Barmherzigkeit nichts zum Verzeihen übriglassen; oder schließlich, man dürfe keine verzeihlichen Sünden bekennen: der sei mit dem Anathema belegt ${ }^{11}$.

Es wäre im Blick auf das Trienter Konzil viel zu ergänzen zu den traditionsgeschichtlichen Hintergründen dieser Lehrformulierungen, die sich vordergründig als Gegenrede gegen die CA in zwei Themenbereichen lesen lassen: (1) nicht zwei (Reue und Glauben), vielmehr drei (Reue, Bekenntnis und Genugtuung) menschliche Anteile an einem wirksamen Bußgeschehen; (2) das vollständige Bekenntnis aller schweren Sünden ist erforderlich.

\subsection{Beichte und Buße als Thema der ökumenisch motivierten Veröffentlichungen zur Confessio Augustana}

In nicht wenigen Veröffentlichungen, die sich seit Mitte der 70er Jahre des 20. Jahrhunderts mit der Frage nach einer möglichen ökumenischen Anerkennung der CA befasst haben, wird der Themenkreis Beichte und Buße explizit angesprochen. Dabei richtete sich die Aufmerksamkeit vor allem auf die (scheinbare) Schwierigkeit, dass in CA 12 von (nur) zwei Teilen der Buße die Rede ist (Reue und Glaube), während das Trienter Konzil an den drei Teilen der Buße

$10 \mathrm{DH}, 1704$.

$11 \mathrm{DH}, 1707$. 
(Reue, Bekenntnis und Genugtuung) festhält. ${ }^{12}$ Die Analyse der Quellentexte belegt, dass es bereits in den Ausschüssen des Augsburger Reichstags 1530 möglich war, sich darauf zu verständigen. Grund dieser Kontroverse sei ein Missverständnis: Die reformatorische Theologie spricht von den inneren Voraussetzungen der Wirksamkeit der Buße durch Reue und Glaube, während die Konzilsväter in Trient an der institutionellen kirchlichen Ordnung festhalten wollten, die in ihrem äußeren Vollzug die Bekundung der Reue, die Bereitschaft zu einem Bekenntnis sowie (in der Folge der erfahrenen Absolution) den Vorsatz zur Aussöhnung mit der beschädigten Lebenswirklichkeit voraussetzte. Viel seltener war bisher im Blick, welche bleibende theologische Relevanz das reformatorische Augenmerk auf die Schwierigkeit eines vollständigen Sündenbekenntnisses hat.

Bereits im Kontext der Bemühungen um eine ökumenische Anerkennung der CA vor 1980 wurde die gemeinsam erkannte seelsorgliche Relevanz der Thematik Beichte und Buße als eine Perspektive der Hoffnung betrachtet. Hans Jorissen hat seinen 1977 veröffentlichten Beitrag mit dem Gedanken beschlossen:

Das kirchliche Beichtgebot hat die gegenwärtige Krise des Bußsakramentes in der katholischen Kirche ebenso wenig verhindern können wie Luthers Optimismus sich erfüllt hat, wenn nur recht von der Beichte gelehrt werde, „so künnde man Lust und Liebe dazu machen, daß die Leut erzukämen und uns nachliefen, mehr denn wir gerne hätten“. Die damit angezeigte Gemeinsamkeit einer pastoralen Aufgabe könnte ein Weg der Verständigung sein. $^{13}$

Ich folge dieser Gedankenspur und frage im Fortgang nach den Lebensbezügen der Thematik Beichte und Buße heute.

\section{Beichte und Buße in der Gegenwart}

Dichterinnen und Dichter nehmen das Leben deutend wahr. Scheinbar Altvertrautes wird in der literarischen Betrachtung zunächst fremd und kommt dann ganz neu nahe. Ein Gedicht von Gottfried Benn ${ }^{14}$ ist mit dem Titel Nur zwei Dinge überschrieben:

12 Vgl. zu dieser Thematik auch die Bemühungen um eine Sichtung der Konvergenzen und der im 16. Jahrhundert verbliebenen Kontroversen in der Bußtheologie in: Karl Lehmann/Wolfhart Pannenberg (Hg.), Lehrverurteilungen - kirchentrennend? Rechtfertigung, Sakramente und Amt im Zeitalter der Reformation und heute, Bd. 1, Freiburg i.B./Göttingen 1986, 63-72.

13 Jorissen, Bußlehre, 150. Das Zitat im Zitat ist aus Martin Luther, Großer Katechismus: BSELK, 1162 (dort in revidierter Sprachgestalt).

14 Gottfried Benn, Nur zwei Dinge (1953), in: ders., Sämtliche Gedichte, Stuttgart 1998, 320. 


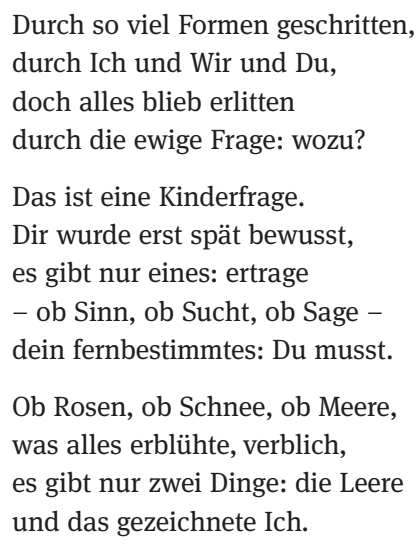

Vielerlei Gestalt nimmt das Leben in Gemeinschaft an: Ein Ich lebt mit einem Du und immer auch im Wir. In allem bleibt die Frage nach dem Grund und dem Sinn dessen, was ist und sich beständig wandelt. Das Leben als nicht frei gewählte Auflage erfüllen, ist eine zu ergreifende Möglichkeit: Du musst, sagt der Dichter. Doch das Wissen um Endlichkeit und Sterblichkeit des Schönen legen sich schwer auf das Gemüt: Was alles erblühte, verblich. Nur zwei Dinge bleiben: die Leere und das gezeichnete Ich.

In der Rede von dem durch das Leben gezeichneten Ich, das in sich wandelnden Formen der Gemeinschaft lebt, wie ein Kind nach den Zusammenhängen fragt und um den Daseinssinn angesichts des Todes und der Sünde ringt, lässt sich ein Menschenbild erkennen, dessen Wesenszüge als Voraussetzung für die Möglichkeit, Erleichterung des Lebens erreichen zu können, gelten: Sozialfähigkeit, Selbstreflexion, Suche nach Verstehen, Wissen um die Sterblichkeit sowie Bereitschaft zum treuen Dienst kennzeichnen den geschilderten Menschen. Das durch die Lebensgeschichte gezeichnete Ich muss jedoch nicht in die Leere fallen. Es gibt Wege erfüllender Deutung und Verwandlung des Daseins. Das Leben ist nicht nur einfach zu ertragen. Es gibt Hoffnung auf eine Erleichterung des Lebens. Ohne diese Aussicht wäre nicht zu begründen, dass Menschen ihr immer auch schuldbeladenes Leben vor Anderen thematisieren.

\subsection{Sündenbekenntnis}

\subsubsection{Biographisches Erzählen - von vielen erwünscht}

Viele Menschen suchen heute nach Gelegenheiten, die Zusammenhänge ihres Lebens zu thematisieren. Das biographische Erzählen ist in der Alltagskommu- 
nikation weit verbreitet. Die Medien tragen dem wachsenden Interesse der Menschen Rechnung, sich in eigenen Konflikten an der Weise zu orientieren, wie andere Menschen das Leben mit seinen Beeinträchtigungen bestehen. Viele autobiographische Schriften erscheinen auf dem Buchmarkt. Die wissenschaftliche Disziplin der Biographieforschung untersucht die Bedeutung des lebensgeschichtlichen Erzählens für die menschliche Identitätsfindung. Zu den primären Funktionen, die biographische Bemerkungen im Alltag haben, zählen zum einen die rechtfertigende Begründung (ein apologetisches Moment), die Selbstvergewisserung (ein anamnetisches Geschehen) und schließlich die Handlungsorientierung (ein prognostischer Zug). Auffällig ist bei dieser Zusammenstellung das Vorkommen der dreifachen Zeitstruktur menschlicher Wirklichkeitswahrnehmung: Menschen erinnern, gegenwärtigen und planen ihr Leben, und all dies geschieht mehr oder weniger bewusst. Die Wahrnehmung der eigenen Zeitlichkeit wirkt sich auf die Gestalt der Thematisierung des widerfahrenen Lebens aus. Das Wissen um die Endlichkeit des Lebens und das Erspüren der Besonderheit bestimmter Zeiten gelten als wichtige Motivationen für die Bereitschaft von Menschen, von sich zu erzählen. In Krisenzeiten kommen größere Zeiträume in den Blick der von sich Erzählenden. Wenn Diskontinuitäten im Lebenslauf eintreten, wenn Entscheidungen anstehen oder ein Abschied zu bestehen ist, dann erhöht sich die Bereitschaft, autobiographisch zu erzählen. Bei der erzählerischen Selbstthematisierung entwerfen die einzelnen Menschen ein Konzept ihres Lebens. Der Lieferant des Materials ist das Gedächtnis, das wichtig Erscheinendes erinnert, eine Verkettung von Ereignissen herstellt und Deutungen vornimmt. Beim biographischen Erzählen wird die unverwechselbare Eigenart des einzelnen menschlichen Lebens offenkundig. Zugleich tritt aber auch die unaufhebbare Verbundenheit der einzelnen mit den Anderen in Erscheinung. Die Beschreibung des eigenen Lebens kommt ohne die Beschreibung des Lebens der Anderen nicht aus. Gelebtes und Erlittenes verbinden sich zu einer unverwechselbaren Geschichte mit den Anderen und durch die Anderen. Die Mitlebenden sind beim Erzählen gegenwärtig. Beim Nachdenken über die Strukturen des biographischen Erzählens treten die Temporalität, die Subjektivität und die Sozialität menschlicher Lebenswahrnehmung vor Augen.

Es erscheint mir wichtig, die Forderung des Trienter Konzils nach einem vollständigen Sündenbekenntnis unter den geschilderten Aspekten zu betrachten. ${ }^{15}$ Es kann dabei nicht um einen Leistungswettbewerb in der Differenzierung der Erinnerungen gehen, auch nicht um die Förderung der Scham im Aussprechen eines Sachverhalts, schon gar nicht um die Befriedigung der möglichen Neugier des

15 Vgl. DH, 1707. 
Gegenübers. Es geht vielmehr um die durch das Einzelbekenntnis ermöglichte, situativ konkrete Lebenshilfe.

\subsubsection{Einzelheiten im Leben und deren Hintergründe}

Viele Menschen erfahren Lebenssituationen, in denen es wichtig ist, mit einem Menschen in ein Gespräch zu kommen, der die Gabe hat, in geduldiger und kundiger Weise Einsicht in die unheilstiftenden Lebenszusammenhänge zu vermitteln und Wege der Aussöhnung mit dem gewordenen Dasein aufzuzeigen. Einzelne Taten haben oft tiefliegende Motivationshorizonte, die es zu lichten gilt. Erst ein solches einfühlendes Verstehen der Kontexte menschlichen Handelns birgt die Aussicht auf dauerhafte Besserung.

Das Charisma der Heilung von Lebenswunden ist nicht jedem gegeben. Persönliche Reife ist erforderlich für eine fruchtbare Wirksamkeit in der Begegnung mit Menschen, die unter ihrer Schuld leiden. Bestehende Begabungen für diesen Dienst können durch eine entsprechende fachliche Qualifizierung gefördert werden. In vielen großen Städten und an Wallfahrtsorten gibt es Beichtzentren, an denen Priester anzutreffen sind, die sich in jahrelanger Weiterbildung auf ihren Dienst vorbereitet haben. In dem Maße, wie Menschen das Gespräch über ihr mit Schuld beladenes Lebens als erlösend und befreiend erleben, werden sie die Orte aufsuchen, an denen ihnen dieses widerfährt. In diesem Geschehen wird von Menschen als hoch bedeutsam erfahren, ob sie ihr personales Gegenüber als authentisch, zugewandt und wohlwollend erleben.

\subsubsection{Das Bekenntnis als vielfältiges Phänomen - historisch betrachtet}

In historischer Betrachtung ist das Sündenbekenntnis ein vielfältig sich wandelndes Phänomen. Ein Kennzeichen des Kanonischen Bußwesens im Altertum war sein Öffentlichkeits-Charakter: Der Ausschluss aus der Gemeinde trat als Folge der schweren Sünde ein. Die als gottesdienstliche Feier gestaltete, an das Schuldbekenntnis sich anschließende Aufnahme des Sünders oder der Sünderin in den Büßerstand galt als erste Stufe im langwierigen Prozess der Wiederaufnahme in die Gemeinde. In auch äußerlich erkennbarer, zeichenhaft gelebter Weise bekundeten die Umkehrwilligen durch zum Teil langwierige, das Leben spürbar verändernde Bußübungen ihre neue Gesinnung. Das Sündenbekenntnis war in der Zeit der öffentlichen Buße weniger bedeutsam; alle Angehörigen der kleinen christlichen Gemeinden wussten ohnehin von den Vorfällen. Entscheidend war zunächst die Bereitschaft zur tätigen Umkehr. Das Sündenbekenntnis 
war das Initialgeschehen für ein öffentliches Verfahren, um deren Dringlichkeit die Gemeinde bereits wusste und es nur einmal im Leben zugestand.

Nach der Konstantinischen Wende und mit der Entwicklung des Christentums zur vorherrschenden Gestalt der Religiosität änderten sich auch die Formen der Versöhnungsfeiern. Im frühen Mittelalter bildete sich eine häufig zu vollziehende, auch im Falle kleinerer Verfehlungen sinnvoll erscheinende Buße heraus, bei der das Sündenbekenntnis und die Bußauflage in Gestalt eines festen Bußtarifs vorrangig bedeutsam waren (sogenanntes Tarifbußwesen). Diese Bußgestalt war ein Erbe des klösterlichen Gemeinschaftslebens und gewann im Zuge der Mission iroschottischer Mönche auf dem europäischen Festland ab dem 7./8. Jahrhundert zunehmend an Bedeutung. Die neue Bußgestalt unterschied sich von der bis dahin geübten in der Häufigkeit (unbeschränkte Wiederholbarkeit), im Zeitpunkt (kein Aufschub in die Sterbestunde aus Angst vor der sonst möglicherweise zu frühzeitig ergriffenen einmaligen Bußmöglichkeit und den harten, dauerhaft belastenden Bußauflagen), im existentiellen Bezug (auch leichtere Sünden), im Verfahren (ohne Öffentlichkeit der Gemeinde, veränderte Bedeutung des Sündenbekenntnisses als Tat der Selbstbeschämung und zur Festlegung adäquater Bußtarife) und im angenommenen Grund der Wirksamkeit (Ausübung von Gott ermöglichter geistlicher Vollmacht der Beichtväter). Das Schuldbekenntnis war nun primär die Grundlage für die Bemessung einer der Schwere der Schuld entsprechenden Bußleistung, bei deren Festlegung detaillierte Angaben in Bußbüchern Hilfestellung gaben.

Die Wandlung der Sinngebung des Schuldeingeständnisses von einem Geschehen zunächst mit öffentlich erkennbarer sozial-ekklesialer Bedeutung zu einer Voraussetzung für ein Gespräch im Rahmen der geistlichen Begleitung eines Menschen, die im ausgehenden Altertum einsetzte, hat sich trotz mancher Reformvorhaben bis heute durchgehalten.

\subsection{Innerliches und Äußerliches}

Reue, Bekenntnis und Genugtuung (materia) sowie Absolution (forma) haben sich in der wechselvollen Geschichte der Feiergestalt der Buße als Konstanten erwiesen, für die sich jedoch eine Variabilität aufzeigen lässt, die nachdenklich stimmen kann. Mein Anliegen dabei ist vor allem, sozial-anthropologische Erkenntnisse in ein Gespräch mit der christlichen Tradition zu bringen.

Von mir vorausgesetzt wird dabei folgende Annahme: Die Vielgestalt der kirchlichen Feierformen im Geschehen der menschlichen Umkehr und der von Gott verheißenen Versöhnung sollte und könnte der Vielgestalt der menschlichen Lebenssituationen entsprechen. Es lassen sich meines Erachtens insbesondere drei eigenartige Gestalten der christlichen Buße unterscheiden, die jeweils eine spezi- 
fische menschliche Situation aufgreifen: (1) Die Feier der Wiederversöhnung mit Gott durch die Wiederaufnahme in die eucharistiefeiernde Gemeinschaft im Falle einer schwerwiegenden Infragestellung des in der Taufe gegebenen Versprechens, im Geist Jesu Christi ein Leben der Liebe gestalten zu wollen. (2) Die von kompetenten, entsprechend ausgebildeten Gesprächspartnern ermöglichte geistliche Begleitung als ein lebenslanger Prozess der Reflexion auf die dunklen Seiten der eigenen Existenz in der Hoffnung auf Lichtung der Lebenszusammenhänge. (3) Gemeinschaftliche Feiern der Buße, in denen Menschen, die sich wechselseitig auch im alltäglichen Leben als bedeutsam erfahren, die berechtigte Erwartungen aneinander hatten und die enttäuscht und verletzt sind durch die Mitlebenden, einander offen ihre Schuld eingestehen und nach neuen Wegen des versöhnten Miteinanders suchen. Bei diesen drei Formen behalten die Einzelaspekte der kirchlichen Versöhnungsfeiern allesamt ihre Bedeutung, hilfreich wäre es jedoch, sie entsprechend der jeweiligen Sozialgestalt, in der eine Schuldverstrickung wirksam wird, zu differenzieren.

\subsubsection{Leidvolle Folgen des eigenen Handelns erkennen}

Die Reue erwächst aus der bitteren Erkenntnis der leidvollen Folgen des eigenen Handelns. Dabei lassen sich Folgen im Blick auf die eigene Lebensgestaltung und Folgen für das Leben anderer Menschen unterscheiden. Als Kriterium in der Frage, wann eine Folge beklagenswert ist, kann die Achtsamkeit auf Formen der Lebensbeeinträchtigung oder gar der Beraubung des Lebens anderer Menschen gelten. Doch ist es nicht immer leicht vorauszusehen, welche Wirksamkeit eine Tat nachhaltig entfalten wird.

Als personale, frei begangene und im geschöpflichen Beziehungsgefüge wirksame Sünde bezeichnet die Theologie auf der Basis der biblischen Überlieferung einen von Menschen vollzogenen Bruch der Gemeinschaft mit Gott, durch den auch die Daseinsmöglichkeiten anderer Menschen beeinträchtigt werden. Dabei war in den älteren biblischen Schriften zunächst ohne Bedeutung, ob eine Tat willentlich oder unabsichtlich geschah, entscheidend war die eintretende leidvolle Folge. Der Gedanke, dass sich die Sünde als Sünde in der auch erfahrbaren Schädigung des Lebens erweist, bleibt auch in den ethischen Weisungen Jesu im Grundsatz erhalten: Nicht etwa erst die Tat des Tötens schädigt das Leben des Mitmenschen, sondern bereits jede im Herzen begangene Anfeindung (Mt 5,21f.). Das Zehngebot, der Dekalog, schärft als von Gott unbedingt geforderte Lebensregel ein, die Daseinsrechte der Mitgeschöpfe zu schützen: niemandem das Leben zu nehmen durch treuloses Verhalten, durch absichtliches Töten, durch den Bruch der familiären 
Gemeinschaft, durch eine Falschaussage vor Gericht oder den Raub des für die Mitmenschen lebensnotwendigen Eigentums.

Das Wahrnehmen der Schädigung des allen Geschöpfen von Gott geschenkten Lebens führt in die Reue. Die Formen dieser Schädigung können sehr unterschiedlich sein. Die leidvollen Auswirkungen können leichter, schwerer oder gar nicht mehr zu verhindern sein. Differenzierende Aufmerksamkeit auf die konkrete Last, die Menschen einander sind, ist eine Bedingung der Möglichkeit, nach Wegen der Erleichterung des Lebens zu suchen. Dabei sind die Lebenswünsche aller in einem Handlungszusammenhang mitwirkenden Menschen $\mathrm{zu}$ bedenken.

\subsubsection{Um die eigene Verwobenheit in die Zusammenhänge wissen}

Nicht zuletzt das derzeit vielbesprochene Stichwort „Globalisierung“ hat die Weltgemeinschaft darauf aufmerksam gemacht, welche auch unheilvollen Verbindungen zwischen ökonomischen, ökologischen und politischen Bereichen bestehen. Ganz konkret ist die Not hungernder, dürstender sowie von Epidemien, Krankheiten und Naturkatastrophen geplagter Menschen in einzelnen Regionen der Erde - nachhaltig in Afrika und Südostasien. Menschen in Not bekommen Gesichter durch die weltweite Berichterstattung. In der globalisierten Welt kommen alle sich nahe. Die Not erbringt Nähe - jenseits aller Grenzen von Nationalität oder Stammeszugehörigkeit. Was wäre zu tun, tatkräftig die Güter der Schöpfung gerechter zu verteilen? Ohnmacht angesichts all der weltweiten Formen der in ungleicher Weise einzelnen Erdregionen zugemuteten Lebensentbehrungen prägt das Empfinden vieler Betrachter. Welche Formen der Reue nehmen diese Erkenntnisse angemessen auf? Müssten oder könnten wir in den christlichen Gemeinden eine Prüfung unserer Lebensverhältnisse anregen und danach fragen, ob alle erworbenen Güter angesichts der Not weltweit erforderlich wären? Schwer vorstellbar ist dies und unvertraut. Zugleich erscheint es als unangemessen, einzig ein Fehlverhalten im Bereich der personalen Beziehungen - eine Wiederheirat Geschiedener etwa - als eine solche Tat zu betrachten, deren Unverträglichkeit mit den Weisungen Gottes zu einem Ausschluss aus der eucharistischen Gemeinschaft führt.

Das Wissen darum, in schwer löslichen Verstrickungen gefangen zu sein, ist vielen Menschen sehr vertraut. Es fällt daher schwer, sich der Rede von der Sünde zu öffnen, da unter diesem Begriff zumeist ein einzelner absichtlich geschehener Fehltritt mit konkreten Folgewirkungen verstanden wird. Was aber könnte es heißen, von der Macht der Sünde in Christus Jesus befreit worden zu sein und diese Wirklichkeit in der Taufe zu feiern? Von der Gestalt der personalen Sünde und der Frage nach ihrem Grund unterscheidet die theologische Tradition die Frage nach 
dem vor-personalen Bösen, das Menschen vorgängig zu ihrer eigenen Entscheidung prägt, beeinflusst und in den Entfaltungsmöglichkeiten beschränkt. Die Rede von der Erbsünde meint Formen des Bösen, die einmal durch personale Sündentaten verursacht wurden, dann aber weiterwirkten und strukturelle Beeinträchtigungen erzeugten.

Die hohe Bereitschaft nicht weniger Menschen heute, das eigene Leben in seinen gewordenen Zusammenhängen zu bedenken, spiegelt sich auch in der gewachsenen gesellschaftlichen Relevanz psychotherapeutischer Arbeit. Die in aller Regel auch mit der Methode des biographischen Erzählens arbeitenden, in ihren anthropologischen Grundannahmen recht unterschiedlichen Schulrichtungen der Psychologie konvergieren gegenwärtig in der Überzeugung, dass die Befreiung eines Menschen von Lebensbeeinträchtigungen, die von ihm als unheil erfahren werden, nur im Gesamt der Beziehungswirklichkeit dieses Menschen gelingen kann. Systemisch denkende und arbeitende Zweige der Psychotherapieforschung, die auf die Verwobenheit einzelner Lebensgeschichten in das Gesamt der generationenübergreifenden Familienerfahrungen hinweisen, erfahren derzeit Wertschätzung. Beachtung findet zudem die eigene Stärke gruppentherapeutischer Arbeit: Das Wissen darum, dass auch andere Menschen die Grenzen der Erfüllung menschlicher Sehnsucht nach einem glückenden Leben erleiden, tröstet und ermutigt dazu, miteinander nach erprobten Wegen der Besserung Ausschau zu halten.

\subsubsection{Die Wurzelsünden (immer wieder) erkennen}

In jüngerer Zeit hat die alte christliche Tradition, die Wurzeln des eigenen sündigen Handelns zu erkennen und in ihren Folgewirkungen $\mathrm{zu}$ bedenken, wieder an Bedeutung gewonnen. Es gibt Pfarrgemeinden, die in Bußgottesdiensten die Mitfeiernden einladen, dem Priester im Einzelbekenntnis das Wichtigste, die Grundwurzel ihrer Einzelsünden zu sagen. Diese Praxis greift die aus der geistlichen Tradition des östlichen Mönchtums stammende Vorstellung von den Wurzelsünden auf, die in der überlieferten Gestalt als Gula (Unmäßigkeit, Völlerei), Luxuria (Unzucht, Unkeuschheit), Avaritia (Geiz, Habsucht), Ira (Zorn), Acedia (Trägheit, Lauheit), Invidia (Ruhmsucht, Neid) und Superbia (Hochmut, Stolz) bestimmt werden. ${ }^{16}$ Es entspricht dem reichen geistlichen Erfahrungsschatz der Kirche, diese Grundantriebe, die durch eine Isolation zu sündigen Beweg-

16 Vgl. Michael Schneider, Aus den Quellen der Wüste. Die Bedeutung der frühen Mönchsväter für eine Spiritualität heute, Einsiedeln 1987. 
gründen menschlichen Handelns werden können, zu bedenken, und einzelne Taten in Bezug auf diese zu betrachten. Meines Erachtens hilft diese Anstrengung dabei, eine bloße Aufzählung einzelner Sündentaten - dazu noch in der Angst, etwas vergessen haben zu können und dadurch die Wirksamkeit des Zuspruchs der Versöhnung Gottes zu gefährden - zu vermeiden. Auf unterschiedliche Weise tragen wohl alle Menschen diese Wurzelsünden in sich. Der Blick auf die konkreten Lebensumstände, in denen sie sich leidvoll auswirken, hilft bei dem Bemühen, die Last des Lebens zu erleichtern.

\section{Reflexionen}

Eine Verbindung zwischen dem menschlichen Leben und der christlichen Buße zu suchen, war der reformatorischen Tradition nicht fremd - im Gegenteil. „Als unser Herr und Meister sagte: ,Tut Buße‘ (...), wollte er, dass das ganze Leben der Gläubigen Buße sei“17. Diese erste der 95 Thesen von Martin Luther, die er - wie er eingangs ausdrücklich sagt - ,aus Liebe zur Wahrheit“ formuliert hat, bringt ins Wort, was als das Grundanliegen der reformatorischen Bewegung nicht nur im Blick auf das Bußsakrament gelten kann: Personale Authentizität wird angestrebt, Echtheit im Umkehrwillen gesucht, nicht Angst vor Strafen soll das Handeln leiten, Verbundenheit von Gottesdienst und Alltag wird vorausgesetzt - kurz: keine äußere Werkerei, sondern innere Entschiedenheit aus bewusstem Glauben heraus soll das Bußwesen prägen.

Der einzelne Augenblick - erlebt in der gefeierten Gestalt der Vergebungszusage Gottes in der gottesdienstlichen Feier - steht in enger Verbindung zu den vielen Momenten des tagtäglichen Lebens, das immer wieder vor Augen führt, wie begrenzt der Wille zur Umkehr im konkreten Handeln je eingelöst werden kann. Die Vorsätze sind immer wieder groß. Die gelebte Wirklichkeit ruft auf zur Barmherzigkeit mit dem eigenen Leben. Vielleicht können Menschen am schwersten mit ihren eigenen Sünden umgehen und sich selbst annehmen. Auch dann ist es ein Hoffnungszeichen, wenn wenigstens kleine Schritte in die gewünschte Richtung gelingen.

Martin Luther sah bei sich selbst - und nicht nur bei anderen Getauften - Tag für Tag die Notwendigkeit, zur Besinnung auf das eigene Leben zu ermahnen. Er blieb bei aller Schärfe im Wort, die zu Teilen auch das Ergebnis erlittener Verletzungen war, ein zutiefst reumütiger Mensch. Die christlichen Traditionen sind reich an liturgischen Formen, in denen Schuldbekenntnisse in allgemeiner Form vorge-

17 WA 1, 233. 
tragen werden. Jeder und jede Beteiligte kann eigene Lebenssituationen in das Gesagte hineindenken. Das Bekenntnis zur sogenannten „Allgemeinen Schuld“ verbindet die westlichen Konfessionsgemeinschaften in ihren eucharistischen Liturgien. Gemeint ist damit eine öffentlich bekundete Zustimmung zu der Wahrnehmung, mit dem eigenen Leben hinter den Anforderungen des Evangeliums Jesu Christi immer wieder zurückzubleiben. Unnütz kann diese geistliche Übung nicht sein.

Ist das allgemeine Schuldbekenntnis eine unzulässige Konkurrenz zur Beichte? In der Geschichte der Ökumene haben die christlichen Kirchen miteinander und voneinander gelernt, die Vielfalt der Formen gottesdienstlicher Feiern als einen Reichtum zu betrachten: Es gibt Lebenssituationen, die es nicht notwendig angeraten erscheinen lassen, Gottes Versöhnungszusage als Wort an einen einzelnen Menschen zu erbitten. Wege der geistlichen Begleitung, die nicht immer mit einem persönlichen Bekenntnis und einem sakramentalen Vergebungszuspruch abschließen, stehen im Lebensalltag jedem Menschen offen. Die reformatorische Bewegung hat dazu beigetragen, das Leben der getauften Menschen immerzu auch unter dem Aspekt der bleibenden Sündigkeit zu betrachten. Jeder Tagesrückblick am Abend gibt Anlass dazu, neben dem Gelungenen und positiv Widerfahrenen auch die eigenen Versäumnisse oder die missglückten Taten wahrzunehmen.

Nicht nur aus reformatorischer Sicht ist das Bußgeschehen in enger Beziehung zur Taufe zu deuten. Gemeinsam betrachten die christlichen Konfessiongemeinschaften die Taufe als die Feier der Lebenswende im Bekenntnis zu Jesus Christus. Mit der neutestamentlich überlieferten paulinischen Tauftheologie (vgl. vor allem Röm 5 und Röm 6) stimmen alle christlichen Konfessionsgemeinschaften darin überein, dass in der Taufe das grundlegende Umkehrgeschehen gegeben ist: Der alte Mensch, der sündige Adam, wird reingewaschen von der Sünde und ersteht verwandelt zur Hoffnung auf unverlierbares Leben. Rein aus Gnade gewährt Gott diese Gabe des Lebens den Sünderinnen und Sündern. Aus eigener Kraft allein wäre der Mensch nicht fähig, sich das Leben auf ewig zu bewahren.

Wie bei vielen anderen Fragen ist es auch in der Bußthematik die Ämtertheologie, die das höchste Maß an ökumenischen Differenzen aufweist. Einerseits gilt: Nicht jeder und jede darf den Anspruch erheben, im Namen Gottes Vergebung zuzusagen; eine Beauftragung ist dazu erforderlich - eine Ermächtigung im Namen der Gemeinschaft der Glaubenden; Kenntnisstände und Lebensführung sind dann $\mathrm{zu}$ prüfen. Im besten Fall gibt es auch eine menschliche Begabung $\mathrm{zu}$ diesem schweren Dienst. Und zugleich gibt es gerade im Bereich des Versöhnungsgeschehens eine andere Wahrheit: Es gibt Menschen, die zu einem versöhnlichen Miteinander motivieren, ohne zu diesem Dienst von der Kirche eigens berufen worden zu sein. Die Ausstrahlung dieser Personen wirkt auf andere. In der Traditionsgeschichte sind es vor allem die Mitglieder der geistlichen Gemeinschaften, denen ein 
solches Charisma zugetraut wurde. Martin Luther war - befangen in dieser Position auch angesichts seiner eigenen Biographie - skeptisch gegenüber dem Mönchsstand und der Beichte. Nicht der Blick auf Personen in bestimmten geistlichen Ständen ist auf Zukunft hin entscheidend beim Bußsakrament, vielmehr die Begegnung mit Menschen, die das Evangelium von der Barmherzigkeit Gottes anderen Menschen nahebringen. Vielleicht durchsteht dieses kostbare Sakrament die Dürrezeiten in Gestalten, die manches zu ahnen geben von dem, was möglich ist an Friedensbereitschaft unter unversöhnten Menschen. Die Absolution ist kein Freispruch von der Verantwortung für die leidvollen Sündenfolgen - wohl ein Freispruch von der Möglichkeit, angesichts der Sünde die Gottesgemeinschaft verlieren zu können. Gott bewahrt den Sünder und die Sünderin vor dem ewigen Tod in Ferne zu ihm. 